\title{
CONCERNING DIAGONAL SIMILARITY OF IRREDUCIBLE MATRICES
}

\section{J. HARTFIEL}

Abstract. If $A=\left(a_{i j}\right)$ is an $n \times n$ irreducible matrix, then there are positive numbers $d_{1}, d_{2}, \cdots, d_{n}$ so that $\sum_{k} d_{i} a_{i k} d_{k}^{-1}$ $=\sum_{k} d_{k} a_{k i} d_{i}^{-1}$ for each $i \in\{1,2, \cdots, n\}$. Further, the numbers $d_{1}, d_{2}, \cdots, d_{n}$ are unique up to scalar multiples.

Introduction. Sinkhorn and Knopp in [4] as well as Brualdi, Parter, and Schneider in [1] have shown that if $A$ is a fully indecomposable matrix then there are diagonal matrices $D_{1}$ and $D_{2}$ with positive main diagonals so that $D_{1} A D_{2}$ is doubly stochastic. Further, in each paper $D_{1}$ and $D_{2}$ are shown to be unique up to scalar multiples.

In this paper we prove what is considered the analogue of the above result in terms of irreducible matrices. That is we show that if $A$ is an $n \times n$ irreducible matrix then there is a diagonal matrix $D$, with positive main diagonal, so that

$$
\sum_{k} d_{i} a_{i k} d_{k}^{-1}=\sum_{k} d_{k} a_{k i} d_{i}^{-1}
$$

for each $i \in\{1,2, \cdots, n\}$. Further we show that $D$ is unique up to scalar multiples.

Definitions and notation. Let $n \geqq 2$ be an integer. Let $N=\{1,2, \cdots, n\}$. An $n \times n$ nonnegative matrix $A$ is said to be reducible if there is a permutation matrix $P$ so that

$$
P A P^{T}=\left(\begin{array}{ll}
A_{1} & 0 \\
B & A_{2}
\end{array}\right)
$$

where $A_{1}$ and $A_{2}$ are square. If $A$ is not reducible we say that $A$ is irreducible. By agreement each $1 \times 1$ matrix is irreducible. Denote

$$
m(A)=\min _{\phi \neq M_{\Phi} N} \sum_{i \in M ; j \notin M} a_{i j}, \quad M(A)=\sum_{i \in N ; j \in N} a_{i j} .
$$

Fiedler [2] refers to $m(A)$ as a measure of the irreducibility of $A$. It should be clear that $A$ is irreducible if and only if $m(A)>0$. Let

$\Lambda_{n}=\left\{n \times n\right.$ nonnegative matrices $A$ with $\sum_{k} a_{i k}=\sum_{k} a_{k i}$ for each $\left.i \in N\right\}$

Received by the editors November 6, 1970 .

AMS 1969 subject classifications. Prinary 1530; Secondary 1560.

Key words and phrases. Irreducible, diagonally similar, matrix patterns.

Copyright (c) 1971, American Mathematical Society 
and

$D=\left\{\left(d_{1}, d_{2}, \cdots, d_{n}\right)\right.$ so that $d_{k}>0$

for each $k \in N$, and at least one $\left.d_{k}=1\right\}$.

For $A$ irreducible we denote by $S(A)$ a positive number so that $S(A) \cdot m(A)-M(A)>M(A)$. Further we define

$$
\begin{aligned}
f_{A}\left(d_{1}, d_{2}, \cdots, d_{n}\right) & =\max _{\phi \neq M_{\Phi}}\left|\sum_{i \in M ; j \in N} d_{i} a_{i j} d_{j}^{-1}-\sum_{i \in N ; j \in M} d_{i} a_{i j} d_{j}^{-1}\right| \\
& =\max _{\phi \neq M_{\ddagger}}\left|\sum_{i \in M ; j \notin M} d_{i} a_{i j} d_{j}^{-1}-\sum_{i \notin M ; j \in M} d_{i} a_{i j} d_{j}^{-1}\right|
\end{aligned}
$$

where each $d_{k}>0, k \in N$.

Results.

Lemma 1. If $\left(d_{1}, d_{2}, \cdots, d_{n}\right) \in D$ and $\max _{k \in N} d_{k} \geqq S^{n}(A)$, then

$$
f_{A}\left(d_{1}, d_{2}, \cdots, d_{n}\right)>f_{A}(1,1, \cdots, 1) .
$$

PROOF. Reorder $\left(d_{1}, d_{2}, \cdots, d_{n}\right)$ to $\left(d_{1}^{\prime}, d_{2}^{\prime}, \cdots, d_{n}^{\prime}\right)$ where $d_{1}^{\prime} \geqq d_{2}^{\prime} \geqq \cdots \geqq d_{n}^{\prime}$. Let $s$ denote the smallest positive integer such that $d_{s}^{\prime} / d_{s+1}^{\prime}>S(A)$. That there is such an $s$ follows since if for each $k \in\{1, \cdots, n-1\}$, we have that $d_{k}^{\prime} / d_{k+1}^{\prime} \leqq S(A)$, then $d_{1}^{\prime} / d_{n}^{\prime}$ $=\prod_{k=1}^{n-1}\left(d_{k}^{\prime} / d_{k+1}^{\prime}\right) \leqq S^{n-1}(A)$. But $d_{1}^{\prime} \geqq \cdots \geqq d_{n}^{\prime}$, and some $d_{k}^{\prime}=1$ implies $d_{n}^{\prime} \leqq 1$. Hence $d_{1}^{\prime} \leqq S^{n-1}(A) d_{n}^{\prime} \leqq S^{n-1}(A)$. Since $S(A)>$ $2 M(A) / m(A) \geqq 2, S^{n-1}(A)<S^{n}(A)$. It would therefore follow that $\max _{k}^{\prime} d_{k}=d_{1}^{\prime}<S^{n}(A)$, a contradiction. Let $M=\left\{i_{R} \mid d_{i_{R}}=d_{R}^{\prime}\right.$ for each $R \in\{1,2, \cdots, s\}\}$. Then

$$
\begin{aligned}
f_{A}(1,1, \cdots, 1) & <M(A)<S(A) \cdot m(A)-M(A) \\
& \leqq\left(d_{s}^{\prime}\right)\left(d_{s+1}^{\prime}\right)^{-1} \sum_{i \in M ; j \notin M} a_{i j}-\sum_{i \notin M ; j \in M} a_{i j} \\
& \leqq \sum_{i \in M ; j \notin M} d_{i} a_{i j} d_{j}^{-1}-\sum_{i \notin M ; j \in M} d_{i} a_{i j} d_{j}^{-1} \leqq f_{A}\left(d_{1}, d_{2}, \cdots, d_{n}\right) .
\end{aligned}
$$

LEMmA 2. If $\left(d_{1}, d_{2}, \cdots, d_{n}\right) \in D$ and $\min _{k \in N} d_{k} \leqq S^{-n}(A)$ then

$$
f_{A}\left(d_{1}, d_{2}, \cdots, d_{n}\right)>f_{A}(1,1, \cdots, 1) .
$$

Proof. If for each $k \in\{1, \cdots, n-1\}$ we have that $d_{k}^{\prime} / d_{k+1}^{\prime}$ $\leqq S(A)$, it would follow as in the proof of Lemma 1 that $\min _{k} d_{k}$ $=d_{n}^{\prime}>S^{-n}(A)$. Hence for this case there is also a smallest integer $s$ such that $d_{s}^{\prime} / d_{s+1}^{\prime}>S(A)$. The rest of the proof is identical with that of Lemma 1. 
LEMma 3. $f_{A}\left(\lambda d_{1}, \lambda d_{2}, \cdots, \lambda d_{n}\right)=f_{A}\left(d_{1}, d_{2}, \cdots, d_{n}\right)$ for $\lambda>0$.

Lemma 4. $f_{A}\left(d_{1}, d_{2}, \cdots, d_{n}\right)$ achieves a minimum for some $\left(d_{1}\right.$, $\left.d_{2}, \cdots, d_{n}\right) \in D$.

Proof. Since $S(A)>2,(1,1, \cdots, 1)$ belongs to the compact set $\mathcal{K}=\left\{\left(d_{1}, \cdots, d_{n}\right) \in \mathbb{D} \mid S^{-n}(A) \leqq \min _{k} d_{k} \leqq \max _{k} d_{k} \leqq S^{n}(A)\right\}$. By Lemmas 1 and $2, \operatorname{Inf} D f_{A}=\operatorname{Inf} \mathcal{K} f_{A}$, and the result follows.

Lemma 5. Let $\left(d_{1}, d_{2}, \cdots, d_{n}\right) \in D$. Suppose

$$
f_{A}\left(d_{1}, d_{2}, \cdots, d_{n}\right)=\max _{\phi \neq M_{\ddagger}}\left|\sum_{i \in M ; j \notin M} d_{i} a_{i j} d_{j}^{-1}-\sum_{i \notin M ; j \in M} d_{i} a_{i j} d_{j}^{-1}\right|
$$

is achieved on $M_{0}=\left\{i_{1}, i_{2}, \cdots, i_{s}\right\}$. Then

$$
\left\{\sum_{k} d_{i} a_{i k} d_{k}^{-1}-\sum_{k} d_{k} a_{k i} d_{i}^{-1} \mid i \in M_{0}\right\}
$$

contains no sign changes and $\left\{\sum_{k} d_{i} a_{i k} d_{k}^{-1}-\sum_{k} d_{k} a_{k i} d_{i}^{-1} \mid i \notin M_{0}\right\}$ contains no sign changes.

Proof. Let $M_{0}^{\prime}=\left\{i \in M_{0} \mid \sum_{k} d_{i} a_{i k} d_{k}^{-1}-\sum_{k} d_{k} a_{k i} d_{i}^{-1}>0\right\}$ and $M_{0}^{\prime \prime}$ $=\left\{i \in M_{0} \mid \sum_{k} d_{i} a_{i k} d_{k}^{-1}-\sum_{k} d_{k} a_{k i} d_{i}^{-1}<0\right\}$. If both $M_{0}^{\prime}$ and $M_{0}^{\prime \prime}$ are nonvoid, then we would have

$$
\begin{aligned}
& \sum_{i \in M_{0 ; k} ; k N} d_{i} a_{i k} d_{k}^{-1}- \sum_{i \in M_{0} ; k \in N} d_{k} a_{k i} d_{i}^{-1} \mid \\
&<\max \left[\sum_{i \in M_{0}^{\prime} ; k \in N} d_{i} a_{i k} d_{k}^{-1}-\sum_{i \in M_{0}^{\prime} ; k \in N} d_{k} a_{k i} d_{i}^{-1},\right. \\
&\left.\sum_{i \in M_{0}^{\prime} ; k \in N} d_{k} a_{k i} d_{i}^{-1}-\sum_{i \in M_{0}^{\prime} ; k \in N} d_{i} a_{i k} d_{k}^{-1}\right],
\end{aligned}
$$

a contradiction. The proof of the other half of the lemma is similar.

Theorem 1. If $A$ is irreducible, then there is a

$$
D=\text { diagonal }\left(d_{1}, d_{2}, \cdots, d_{n}\right)
$$

with $\left(d_{1}, d_{2}, \cdots, d_{n}\right) \in D$ so that $D A D^{-1} \in \Lambda_{n}$.

Proof. We first prove the theorem for a positive matrix $A$. Suppose $f_{A}\left(d_{1}, d_{2}, \cdots, d_{n}\right)$ achieves a minimum at $\left(d_{1}^{0}, d_{2}^{0}, \cdots, d_{n}^{0}\right) \in \mathbb{D}$. Suppose 


$$
f_{A}\left(d_{1}^{0}, d_{2}^{0}, \cdots, d_{n}^{0}\right)=\left|\sum_{i \in M_{0} ; j \in N} d_{i}^{0} a_{i j}\left(d_{j}^{0}\right)^{-1}-\sum_{i \in N_{i} ; \in M_{0}} d_{i}^{0} a_{i j}\left(d_{j}^{0}\right)^{-1}\right| .
$$

We shall prove that $f_{A}\left(d_{1}^{0}, \cdots, d_{n}^{0}\right)=0$.

Then $\sum_{i \in M ; j \in N} d_{i}^{0} a_{i j}\left(d_{j}^{0}\right)^{-1}-\sum_{i \in N ; j \in M} d_{i}^{0} a_{i j}\left(d_{j}^{0}\right)^{-1}=0$ for any $M$ where $\phi \neq M \Phi N$. The result follows by taking $M=\{k\}$, for each $k \in\{1, \cdots, n\}$. Hence suppose that $f_{A}\left(d_{1}^{0}, \cdots, d_{n}^{0}\right)>0$. Without loss of generality suppose $d_{i}^{0} a_{i j}\left(d_{j}^{0}\right)^{-1}=b_{i j}$ and $\sum_{k \in N} b_{i k}-\sum_{k \in N} b_{k i}>0$ for each $i \in M_{0}$. Let $M_{1}=\left\{i \mid \sum_{k \in N} b_{i k}=\sum_{k \in N} b_{k i}\right\}$ and $M_{2}=N$ $-\left(M_{0} \cup M_{1}\right)$.

In view of Lemma 5 , it must be that $\sum_{k} b_{i k}-\sum_{k} b_{k i}<0$ for all $i \in M_{2}$. In particular, $M_{2}$ is nonempty.

Consider $\left(d_{1}, d_{2}, \cdots, d_{n}\right)$ defined as follows:

$$
\begin{aligned}
d_{k} & =(1+\epsilon)^{-1} & & \text { if } k \in M_{0}, \\
& =1 & & \text { if } k \in M_{1}, \\
& =(1+\epsilon) & & \text { if } k \in M_{2},
\end{aligned}
$$

Now suppose $M_{1}^{\prime} \subseteq M_{1}$. Then consider

$$
\begin{aligned}
g(\epsilon)= & \sum_{i \in M_{0} \cup M_{1}^{\prime} ; j \in N} d_{i} b_{i j} d_{j}^{-1}-\sum_{i \in N_{;} ; \in M_{0} \cup M_{1}^{\prime}} d_{i} b_{i j} d_{j}^{-1} \\
= & \sum_{i \in M_{0} ; j \in M_{1}}(1+\epsilon)^{-1} b_{i j}+\sum_{i \in M_{0} ; j \in M_{2}}(1+\epsilon)^{-2} b_{i j}-\sum_{i \in M_{1} ; j \in M_{0}}(1+\epsilon) b_{i j} \\
& -\sum_{i \in M_{2} ; j \in M_{0}}(1+\epsilon)^{2} b_{i j}+\sum_{i \in M_{1}^{\prime} ; j \in M_{0}}(1+\epsilon) b_{i j}+\sum_{i \in M_{1}^{\prime} ; \in M_{1}} b_{i j} \\
& +\sum_{i \in M_{1} ; j \in M_{2}}(1+\epsilon)^{-1} b_{i j}-\sum_{i \in M_{0} ; \in \in M_{1}^{\prime}}(1+\epsilon)^{-1} b_{i j} \\
& -\sum_{i \in M_{1} ; j \in M_{1}^{\prime}} b_{i j}-\sum_{i \in M_{2} ; j \in M_{i}^{\prime}}(1+\epsilon) b_{i j} .
\end{aligned}
$$

Hence $g^{\prime}(0)<0$. Therefore there is a number $\epsilon_{1}$ so that, if $0<\epsilon<\epsilon_{1}$,

$$
\max _{M_{1}^{\prime}}\left(\sum_{i \in M_{0} \cup M_{M_{1}^{\prime} ; k \in N}} d_{i} b_{i k} d_{k}^{-1}-\sum_{i \in M_{0} \cup M_{1}^{\prime} ; k \in N} d_{k} b_{k i} d_{i}^{-1}\right)<f_{A}\left(d_{1}^{0}, d_{2}^{0}, \cdots, d_{n}^{0}\right)
$$

and

$$
\sum_{k \in N} d_{i} b_{i k} d_{k}^{-1}-\sum_{k \in N} d_{k} b_{k i} d_{i}^{-1}>0 \quad \text { for } i \in M_{0} .
$$

Similarly, for $M_{1}^{\prime \prime} \subseteq M_{1}$, consider 


$$
\begin{aligned}
h(\epsilon)= & -\sum_{i \in M_{2} \cup_{M_{1}^{\prime \prime} ; j \in N}} d_{i} b_{i j} d_{j}^{-1}+\sum_{i \in N ; j \in M_{2} \cup_{M_{1}^{\prime}}} d_{i} b_{i j} d_{j}^{-1} \\
= & \sum_{i \in M_{0} ; j \in M_{2}}(1+\epsilon)^{-2} b_{i j}+\sum_{i \in M_{1} ; j \in M_{2}}(1+\epsilon)^{-1} b_{i j} \\
& -\sum_{i \in M_{2} ; j \in M_{0}}(1+\epsilon)^{2} b_{i j}-\sum_{i \in M_{2} ; j \in M_{1}}(1+\epsilon) b_{i j} \\
& +\sum_{i \in M_{0} ; j \in M_{1}^{\prime \prime}}(1+\epsilon)^{-1} b_{i j}+\sum_{i \in M_{1} ; j \in M_{1}^{\prime \prime}} b_{i j} \\
& +\sum_{i \in M_{2} ; j \in M_{1}^{\prime \prime}}(1+\epsilon) b_{i j}-\sum_{i \in M_{1}^{\prime \prime} ; j \in M_{0}}(1+\epsilon) b_{i j} \\
& -\sum_{i \in M_{1}^{\prime \prime} ; j \in M_{1}} b_{i j}-\sum_{i \in M_{1}^{\prime \prime} ; j \in M_{2}}(1+\epsilon)^{-1} b_{i j} .
\end{aligned}
$$

Hence $h^{\prime}(0)<0$. Therefore there is a number $\epsilon_{2}$ so that, if $0<\epsilon<\epsilon_{2}$,

$$
\max _{M_{1}^{\prime \prime}}\left(\sum_{i \in M_{2} \cup M_{1}^{\prime \prime} ; k \in N} d_{k} b_{k i} d_{i}^{-1}-\sum_{i \in M_{2} \cup M_{M^{\prime \prime} ; k \in N}} d_{i} b_{i k} d_{k}^{-1}\right)<f_{A}\left(d_{1}^{0}, d_{2}^{0}, \cdots, d_{n}^{0}\right)
$$

and

$$
\sum_{k \in N} d_{k} b_{k i} d_{i}^{-1}-\sum_{k \in N} d_{i} b_{i k} d_{k}^{-1}>0 \quad \text { for } i \in M_{2} .
$$

Therefore for $\epsilon_{3}=\min \left(\epsilon_{1}, \epsilon_{2}\right)$ and $0<\epsilon<\epsilon_{3}$ we have in view of Lemma 5, any set $\bar{M}$ for which

$$
f_{A}\left(d_{1}, \cdots, d_{n}\right)=\left|\sum_{i \in \bar{M} ; j \in N} d_{i} a_{i j} d_{j}^{-1}-\sum_{i \in N ; j \in \bar{M}} d_{i} a_{i j} d_{j}^{-1}\right|
$$

must necessarily be of one of the two forms $M_{0} \cup M_{1}^{\prime}$ or $M_{2} \cup M_{1}^{\prime \prime}$ for some $M_{1}^{\prime} \subseteq M_{1}$ or $M_{1}^{\prime \prime} \subseteq M_{1}$. Therefore

$$
f_{A}\left(d_{1} d_{1}^{0}, d_{2} d_{2}^{0}, \cdots, d_{n} d_{n}^{0}\right)<f_{A}\left(d_{1}^{0}, d_{2}^{0}, \cdots, d_{n}^{0}\right) .
$$

But $\left(1, d_{1}^{-1}\left(d_{1}^{0}\right)^{-1} d_{2} d_{2}^{0}, \cdots, d_{1}^{-1}\left(d_{1}^{0}\right)^{-1} d_{n} d_{n}^{0}\right) \in \mathscr{D}$, and since, by Lemma 3 ,

$$
f\left(1, d_{1}^{-1}\left(d_{1}^{0}\right)^{-1} d_{2} d_{2}^{0}, \cdots, d_{1}^{-1}\left(d_{1}^{0}\right)^{-1} d_{n} d_{n}^{0}\right)=f\left(d_{1} d_{1}^{0}, d_{2} d_{2}^{0}, \cdots, d_{n} d_{n}^{0}\right),
$$

$f_{A}$ would not be minimal at $\left(d_{1}^{0}, \cdots, d_{n}^{0}\right)$. This contradiction shows that $f_{A}\left(d_{1}^{0}, \cdots, d_{n}^{0}\right)=0$ as was to be proven.

Now suppose $A$ is irreducible. Let $A_{m}$ be a positive matrix for each positive integer $m$, and $\lim _{m \rightarrow \infty} A_{m}=A$. Then for each $m$ there is a diagonal matrix $D_{m} \in D$ so that $D_{m} A_{m} D_{m}^{-1} \in \Lambda_{n}$. In the proof of Lemma $4, S^{-n}\left(A_{m}\right) \leqq \min _{k} d_{k}^{(m)} \leqq \max _{k} d_{k}^{(m)} \leqq S^{n}\left(A_{m}\right)$. Prudent choices for each 
$S\left(A_{m}\right)$ indicate that the $d_{k}^{(m)}$ are bounded from above and away from zero as $m \rightarrow \infty$. Hence $\left\{D_{m}\right\}$ has a limit point $D$ and $D$ is nonsingular. Let $\left\{D_{m^{\prime}}\right\}$ be a subsequence of $\left\{D_{m}\right\}$ so that $\lim _{m^{\prime} \rightarrow \infty} D_{m^{\prime}}=D$. Then $\lim _{m^{\prime} \rightarrow \infty} D_{m^{\prime}} A_{m^{\prime}} D_{m^{\prime}}^{-1}=D A D^{-1}$ and the result follows.

CoROllaRy 1. If for some permutation matrix $P, P^{T} A P$ is a direct sum of irreducible matrices then there is a $D=$ diagonal $\left(d_{1}, d_{2}, \cdots, d_{n}\right)$ with $\left(d_{1}, d_{2}, \cdots, d_{n}\right) \in D$ such that $D A D^{-1} \in \Lambda_{n}$.

We might remark here that the result essentially characterizes irreducibility, in the sense that if $A$ is a nonnegative $n \times n$ matrix then there exists a $D=\operatorname{diag}\left(d_{1}, \cdots, d_{n}\right),\left(d_{1}, \cdots, d_{n}\right) \in D$ and $D A D^{-1}$ $\in \Lambda_{n}$, if and only if there exists a permutation matrix $P$ such that $P^{T} A P$ is a direct sum of irreducible matrices. The converse part of this statement follows readily from the normal form of a reducible matrix [3, p. 74].

Theorem 2. If $A$ is irreducible, $A \in \Lambda_{n}, B \in \Lambda_{n}$ and $D$ is a diagonal matrix with positive main diagonal so that $D A D^{-1}=B$, then $A=B$ and $D=\lambda I$ for some $\lambda>0$.

Proof. Without loss of generality we may assume

$$
D=\operatorname{diagonal}\left(d_{1}, d_{2}, \cdots, d_{n}\right) \quad \text { where } d_{1} \geqq d_{2} \geqq \cdots \geqq d_{n} \text {. }
$$

If $d_{1}=d_{n}$ then we are through. If $d_{1} \neq d_{n}$, then let $s$ denote the smallest integer so that $d_{s}>d_{s+1}$. Let $M=\{1,2, \cdots, s\}$. If $i \in M, k \notin M$, $d_{i}>d_{k}$ and so $d_{i} a_{i k} d_{k}^{-1} \geqq a_{i k}$, the last inequality is strict for some $i \in M, k \in M$ due to the irreducibility of $A$. Thus

$$
\sum_{i \in M ; k \notin M} d_{i} a_{i k} d_{k}^{-1}>\sum_{i \in M ; k \notin M} a_{i k}=\sum_{i \notin M ; k \in M} a_{i k} .
$$

Similarly $\sum_{i \notin M ; k \in M} a_{i k}>\sum_{i \notin M ; k \in M} d_{i} a_{i k} d_{k}^{-1}$ and, therefore, $\sum_{i \in M ; k \notin M} d_{i} a_{i k} d_{\mathbf{k}}^{-1}>\sum_{i \notin M ; k \in M} d_{i} a_{i k} d_{k}^{-1}$, i.e.

$$
\sum_{i \in M ; k \in N} d_{i} a_{i k} d_{k}^{-1}>\sum_{i \in N ; k \in M} d_{i} a_{i k} d_{k}^{-1}
$$

which gives us a contradiction. Therefore $d_{1}=d_{n}$ and $D=\lambda I$ for some $\lambda>0$.

Corollary 2. If $A$ is irreducible and $D$ a diagonal matrix with $D A D^{-1} \in \Lambda_{n}$, then $D$ is unique up to a scalar multiple.

Acknowledgement. The author is grateful for the referee's suggestions concerning the presentation of this paper. 


\section{BIBLIOGRAPHY}

1. R. A. Brualdi, S. V. Parter, and H. Schneider, The diagonal equivalence of $a$ nonnegative matrix to a stochastic matrix, J. Math. Anal. Appl. 16 (1966), 31-50. MR 34 \#5844.

2. M. Fiedler, Bounds for eigenvalues of doubly stochastic matrices (submitted).

3. F. R. Gantmaher, The theory of matrices, GITTL, Moscow, 1953; English transl., Vol. 2, Chelsea, New York, 1959. MR 16, 438.

4. R. Sinkhorn and P. Knopp, Concerning nonnegative matrices and doubly stochastic matrices, Pacific J. Math. 21 (1967), 343-348. MR 35 \#1617.

Texas A\&M University, College Station, Texas 77843 